\title{
Nutritional status of cancer patients at dietitian referral
}

\author{
C.M. Lorton ${ }^{1,2}$, E. Barnes ${ }^{3}$, N. Gough ${ }^{4}$, O. Griffin ${ }^{3}$, K. Higgins ${ }^{5}$, S. Kielthy ${ }^{6}$, F. Roulston ${ }^{7}$, \\ G. Stewart ${ }^{8}$ and T.D. Walsh ${ }^{1,2,6}$ \\ ${ }^{1}$ Our Lady's Hospice and Care Services, Dublin, Ireland, ${ }^{2}$ Trinity College Dublin, Dublin, Ireland, ${ }^{3}$ St Vincent's \\ University Hospital, Dublin, Ireland, ${ }^{4}$ Mater Private Mid-Western Radiation Oncology Centre, Limerick, Ireland, \\ ${ }^{5}$ Tallaght Hospital, Dublin, Ireland, ${ }^{6}$ University College Dublin, Dublin, Ireland, ${ }^{7}$ St Luke's Radiation Oncology \\ Network, Dublin, Ireland and ${ }^{8}$ St Vincent's Private Hospital, Dublin, Ireland.
}

Malnutrition and cachexia in cancer are associated with adverse prognosis, reduced tolerance of cancer treatment and poor quality of life ${ }^{(1)}$. Furthermore, malnutrition is associated with increased healthcare costs; early intervention may result in significant savings ${ }^{(2)}$. There is increased emphasis on early recognition of people at risk of malnutrition and in the pre-cachexia stage ${ }^{(1)}$.

This study aimed to provide a snapshot of current practice in Ireland and describe

1. The characteristics of people with cancer at point of dietitian referral

2. Professional opinion on adequacy of referral timing

3. The utility of the 2011 consensus definition ${ }^{(1)}$ to identify cancer cachexia in clinical practice.

This was a prospective observational study. Data was recorded in five Irish hospitals from consecutive referrals to dietitian services of people with cancer. Demographic details, barriers to nutrition and timing of referral were noted. Where possible, the dietitian categorised each patient by stage of cachexia, based on clinical assessment. Information on skeletal muscle depletion was not routinely available. Microsoft Excel (2010) was used for statistical analysis.

Data from 175 patients was included, $59 \%$ males and $57 \%$ inpatients. Treatment was palliative in $55 \%$ of cases. Weight loss was the most common reason for referral $(57 \%)$, followed by poor appetite $(18 \%)$. Routine referral due to diagnosis or treatment took place in $17 \%$. Most $(68 \%)$ had lost $5 \%$ or more of body weight. Two or more barriers to nutrition were present for $71 \%$ of patients, most commonly anorexia, nausea and early satiety. In $31 \%$, the dietitian identified a clear missed opportunity for earlier referral. Cachexia stage was assigned in $87 \%$. Of these, $54 \%$ were thought to be cachectic with $11 \%$ refractory and only $16 \%$ pre-cachectic.

Despite growing literature highlighting the importance of nutritional care in cancer, most patients with cancer were still referred late to a dietitian, with established weight loss and multiple nutritional symptoms. Only one sixth of patients saw a dietitian at the precachectic stage and one third had missed earlier opportunities for referral. Although information on sarcopenia was not available, experienced dietitians in clinical practice felt confident assigning cachexia stage for the majority of patients. It could thus be feasible to use this staging system in clinical practice, even without information on sarcopenia. However, validation against clinical outcomes would be essential.

To our knowledge, this is the first study to describe current practice in referrals to Oncology dietitians in Irish hospitals. It will inform service planning, education and future research in malnutrition and cancer cachexia in Ireland.

1. Fearon K, Strasser F, Anker S, et al. (2011) Definition and classification of cancer cachexia: an international consensus. Lancet Oncol 12, 489-495. 2. Rice N \& Normand C. (2012) The cost associated with disease-related malnutrition in Ireland. Public Health Nutr 15, 1966-1972. 